\title{
Pharmacokinetics-Based
}

\section{Optimization of Phototherapy in}

\section{Neonates Undergoing Treatment for Hyperbilirubinemia}

Dibyajyoti SAIKIA a, Subodh KUMAR ${ }^{b}$, T. VELPANDIANc, A. K. DEORARI ${ }^{d}$, N. R BISWASe, Y. K. GUPTA ${ }^{\mathrm{e}}$

aDepartment of Pharmacology, Noida International Institute of Medical Sciences, Greater Noida, India

bDepartment of Pharmacology, All India Institute of Medical Sciences, Deoghar, India

cDepartment of Ocular Pharmacology and Pharmacy,

All India Institute of Medical Sciences, New Delhi, India

dDepartment of Pediatrics, All India Institute of Medical Sciences, New Delhi, India

eDepartment of Pharmacology, All India Institute of Medical Sciences, New Delhi, India

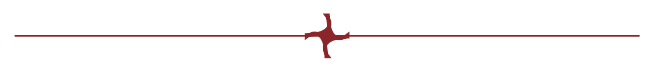

\begin{abstract}
Introduction: Neonatal jaundice results from combined effects of both increased production of bilirubin and decreased hepatic excretory capacity in neonates. Since its discovery, phototherapy is the most widespread treatment used in neonatal jaundice. In this work, we try to search for a relationship between exposure to phototherapy and decrease in serum bilirubin (linearity vs proportionality).

Method: The present research was non-randomized prospective study conducted in the Neonatal Intensive Care Unit (NICU), Department of Paediatrics, AIIMS, New Delhi, and the Department of Pharmacology, AIIMS, New Delhi, India. Subjects were recruited from neonates admitted in NICU AIIMS, which meets our selection criteria. Infants were given a low dose of either phototherapy continuously or phototherapy for the first six hours and a double dose of phototherapy for the next six hours. Samples were collected before the beginning of the study (0 hours) and then at six and 12 hours. Bilirubin concentration was measured using HPLC and (LC-MS/MS).

Results and conclusion: The percentage of reduction during the 6-12-hour interval was compared with that during the 0-6-hour interval if all experimental conditions were kept unchanged. A relationship curve between percentage of reduction and irradiance was created based on the percentage of reduction in serum bilirubin during the 0-6-hour and 0-12-hour intervals. The present study suggests that the relationship between efficacy, as measured by percentage of reduction in serum bilirubin, and irradiance is unlikely to be linear. Collected data are insufficient to clearly distinguish between proportionality and saturation point, considering that the results may be possible with both of these hypotheses.
\end{abstract}

Keywords: neonatal jaundice, phototherapy, hyperbilirubinemia.

Address for correspondence:

Dr. Dibyajyoti Saikia, MBBS (GMCH), MD (AIIMS, New Delhi), Assistant Professor Department of Pharmacology,

Noida International Institute of Medical Sciences, Greater Noida, India

Tel.: +91 7289822218, email: dibyajyotisaikia11@gmail.com

Article received on the $30^{\text {th }}$ of September and accepted for publication on the $8^{\text {th }}$ of December 2021 


\section{INTRODUCTION}

aundice is the most common condition requiring medical attention in newborns $(1,2)$. Bilirubin accumulates in the skin and sclera of newborns, with jaundice producing yellow discoloration. Unconjugated hyperbilirubinemia is a normal transient phase in most neonates. However, if the rise of bilirubin levels is excessive, it may cause neurotoxicity and even death. Neurotoxicity gives rise to a condition known as kernicterus. Prevention of development of neurotoxicity is the primary aim of diagnostic evaluation and need for treatment in neonates with unconjugated hyperbilirubinemia (American Academy of Pediatrics, Subcommittee on Hyperbilirubinemia, 2004).

Physiological jaundice results from combined effects of both increased production of bilirubin and decreased hepatic excretory capacity in neonates $(2,3)$. Bilirubin production is elevated in neonates as neonates have a higher erythrocyte mass than adults. Also, fetal red blood cells have a shorter lifespan (4). A low activity of glucuronyl transferase, an enzyme responsible for the conjugation of bilirubin to glucoronic acid, limits the liver ability to excrete bilirubin $(5,6)$. Bilirubin is the end product of heme catabolism produced in reticuloendothelial system through oxidationreduction reactions. Around $75-80 \%$ of bilirubin is derived from hemoglobin catabolism. Bilirubin is also formed during degradation of myoglobin, cytochromes and oxygenases (7). The rate limiting step during bilirubin formation is the conversion of heme into biliverdin catalyzed by heme oxygenase. While biliverdin is water soluble, due to intramolecular hydrogen bonds, bilirubin is insoluble in water $(8,9)$. Hydrophobic bilirubin binds to albumin and is transported into plasma in this form. The fraction not bound to albumin (free albumin) is able to cross the blood-brain barrier, which is believed to be responsible for its neurotoxicity.

The aim of treatment for neonatal jaundice is to avoid neurotoxicity. Phototherapy is the primary treatment in unconjugated neonatal hyperbilirubinemia (10). Since its discovery in 1950's, phototherapy is now the most widespread treatment which is used in neonates. Efficacy of phototherapy in decreasing bilirubin serum levels is mainly due to structural isomerization of bilirubin into lumirubin. The photoisomers are excre- ted in the bile and urine. Phototherapy is administered by using a light source to irradiate the surface of the neonate using an effective wavelength. The relationship between efficacy and irradiance is a debated topic. There are currently two conflicting theories regarding this issue. The first one was the result of Tan KL's research that suggested the existence of a saturation point at around $40 \mu \mathrm{W} / \mathrm{cm}^{2} / \mathrm{nm}$, after which there was no further increase in either efficacy or irradiance (11). The other theory proposed a linear relationship between these two. Thus, Vandborg PK found a linear relationship between total serum bilirubin and an irradiance level between 20 and $55 \mu \mathrm{W} / \mathrm{cm}^{2} / \mathrm{nm}(12)$.

In this work, a new concept regarding the relationship, termed here as proportionality, has been put forth and a novel approach in study design has been attempted to explore the hypothesized relationship. It is conjectured that, on increasing irradiance, there will be a progressive decline in serum bilirubin in absolute terms $(\mathrm{mg} / \mathrm{dL})$, which will however be constant in terms of proportions (as in percentage decrease). This will result in neither saturation nor a linear relation, but will give a hyperbolic curve. This concept is also supported by Bunsen-Roscoe law of reciprocity for photochemical change (13) as well as fractional product method for additive effect.

\section{Study objectives}

1. Evaluation of the conventional method of phototherapy in the disappearance of serum bilirubin/formation of bilirubin photoisomers over a time period (0, 6 and 12 hours) in infants with neonatal hyperbilirubinemia $(n=15)$;

2. Evaluation of six-hour low intensity phototherapy (20-35 $\left.\mu \mathrm{w} / \mathrm{cm}^{2} / \mathrm{nm}\right)$, followed by doubling the intensity $\left(45-60 \mu \mathrm{w} / \mathrm{cm}^{2} / \mathrm{nm}\right)$ for the next six hours, on disappearance of serum bilirubin/formation of bilirubin photoisomers in babies with neonatal hyperbilirubinemia $(n=15)$;

3. Analysing the possibility of developing an equation to predict the duration of phototherapy based on data provided by the approach described in objective 1 .

\section{MATERIALS AND METHODS}

ite of study
Our research was a non-randomized pro-
spective study conducted in the Neonatal Inten- 
sive Care Unit (NICU), Department of Paediatrics, AlIMS, New Delhi, India, and the Department of Pharmacology, AIIMS, New Delhi, India. The present study was started after obtaining the ethical approval from the Institutional Ethics Sub-Committee (IESC).

Subjects: Patients diagnosed with hyperbilirubinemia, in whom phototherapy was being planned in NICU-B, Department of Paediatrics, AIIMS, New Delhi, India.

Inclusion criteria: Neonates with unconjugated hyperbilirubinemia in whom phototherapy has been considered in accordance with IAP guidelines, age $>34$ weeks and birth weight $>2500$ grams.

Exclusion criteria: Severely ill patients, subjects in whom phototherapy was not considered, those who were already undergoing phototherapy, patients in which exchange transfusion was currently considered or likely to be used soon, and parents/guardians' refusal to give consent.

\section{Recruitment}

Subjects were recruited from neonates admitted in NICU AIIMS, which meets our selection criteria. Consent was taken from parents/legal guardians of neonates in whom phototherapy was considered. After obtaining the consent, samples were taken from neonates in whom phototherapy was started.

Collection of information about study subjects Gestational age assessment was calculated by either the first day of the last menstrual period or the first trimester ultrasound. Newborns were measured for weight as well as length (with an infantometer) and all results were recorded (Table 1). Mothers were asked whether they were breast feeding or not. Any concurrent medication being taken was noted. Phototherapy was given using LED lights in low and double intensity.

\section{Study design}

Regarding the plan of phototherapy for objectives 1 and 3, 15 infants were given low dose phototherapy continuously, and samples were collected before start ( 0 hour) and then at 6 and 12 hours. Test group data were used to explore the possibility of developing an equation to predict the duration and response of phototherapy (objective 3 ). For objective 2 , infants in this group $(n=15)$ were to be given low dose phototherapy for the first six hours and double dose phototherapy for the next six hours. Samples were collected before start ( 0 hours) and then at 6 and 12 hours.

\section{Sampling and analysis}

Under careful aseptic and antiseptic conditions, three samples of $0.5 \mathrm{~mL}$ each were taken just before the start ( 0 hours) and at 6 and 12 hours. Samples were analysed by HPLC and (LC-MS/MS). The present research was aimed to be a pilot study and no sample size calculation was done. The total number of subjects planned to be enrolled was 30 .

\section{Statistical analysis}

Data were entered and further analysed by using using Excel 2010 (Microsoft, Redmond, WA, USA). They were presented as mean (SD) or number (\%). For agreement between predicted and observed values, multiple tests were applied as no single statistical test has been universally accepted. For comparison between groups by t test, the GraphPad InStat Version 3.05 was used. The correlation coefficient and coefficient of determination were calculated using the coefficient calculator available from Social Science Statistics calculator.

\section{RESULTS}

\section{Raseline characteristics}

Ninety blood samples were collected from the 30 subjects recruited for this study. All of them were diagnosed with hyperbilirubinemia and received phototherapy as follows: 15 subjects were given continuous phototherapy for 12 hours and in the remaining 15 ones the irra-

\begin{tabular}{|l|l|l|l|l|l|}
\hline Sex & $\begin{array}{l}\text { Gestational } \\
\text { age (weeks) }\end{array}$ & Term/late preterm & $\begin{array}{l}\text { Birth weight } \\
\text { (grams) }\end{array}$ & $\begin{array}{l}\text { Mode of } \\
\text { delivery }\end{array}$ & $\begin{array}{l}\text { Initial bilirubin } \\
\text { concentration (mg/dL) }\end{array}$ \\
\hline $\begin{array}{l}\text { Male: } 47 \% \\
\text { Female: } 53 \%\end{array}$ & $37 \pm 2$ & $\begin{array}{l}\text { Term*: } 23 \% \\
\text { Late preterm**: } 77 \%\end{array}$ & $2928 \pm 30.8$ & $\begin{array}{l}\text { NVD: } 69 \% \\
\text { CS: } 23 \% \\
\text { Assisted: } 8 \%\end{array}$ & $22.2 \pm 1.9$ \\
\hline
\end{tabular}

TABLE 1.

Subjects' clinical and demographic data 


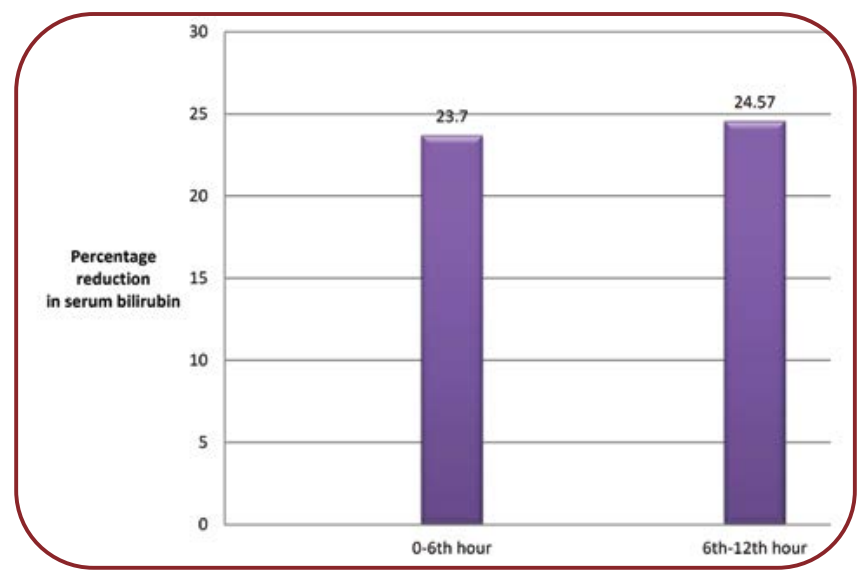

FIGURE 1. Bar diagram showing the percentage of reduction in serum bilirubin during the 0-6- and 6-12-hour intervals

\begin{tabular}{|l|l|l|l|l|l|l|}
\hline & $\begin{array}{l}\text { \% of reduction } \\
\text { during the 0-6- } \\
\text { hour interval }\end{array}$ & $\begin{array}{l}\% \text { of reduction } \\
\text { during the } \\
\text { 0-12-hour } \\
\text { interval }\end{array}$ & $\begin{array}{l}\text { Predicted } \\
\text { reduction } \\
\text { (linearity) }\end{array}$ & $\begin{array}{l}\text { Predicted } \\
\text { reduction } \\
\text { (saturation) }\end{array}$ & $\begin{array}{l}\text { Predicted } \\
\text { reduction } \\
\text { (proportionality) }\end{array}$ & $\begin{array}{l}\text { Actual } \\
\text { reduction } \\
\text { (6 to 12 } \\
\text { hours) }\end{array}$ \\
\hline Mean & 25.21 & 51.5 & 50.43 & 25.21 & 43.61 & 35.61 \\
\hline
\end{tabular}

TABLE 2.

Proportional reduction in serum bilirubin in the doubled irradiance group

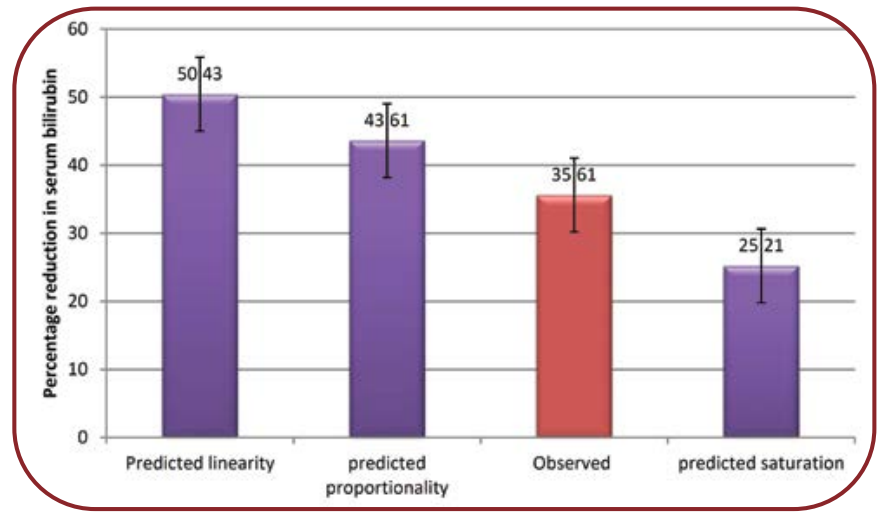

FIGURE 2. Bar diagram showing the mean percentage of reduction (6-12 hours) in serum bilirubin as predicted by linearity, saturation and proportionality along with actual observed percentage of reduction. Error bars represent the standard error of mean

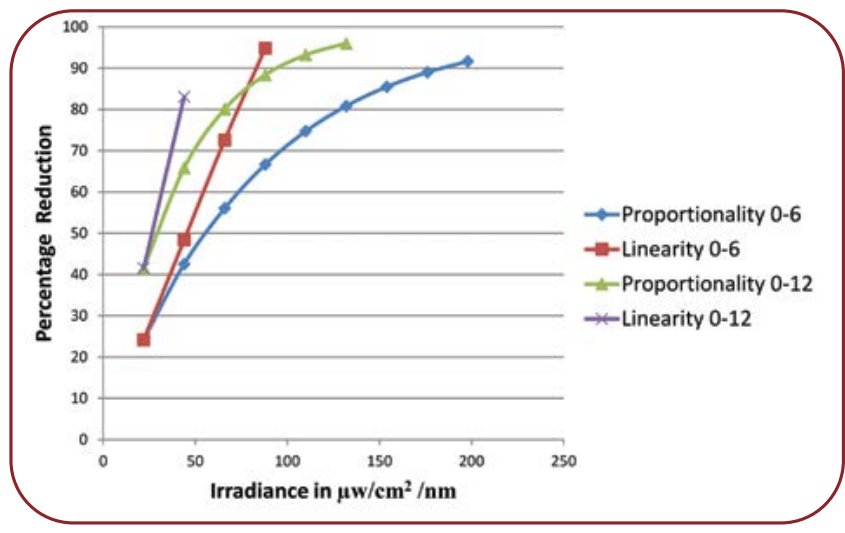

FIGURE 3. Curve derived from actual values of reduction in serum bilirubin during the 0 -6- and 0-12-hour intervals vs irradiance $\left(\mu \mathrm{w} / \mathrm{cm}^{2} / \mathrm{nm}\right)$

diance level was doubled after six hours and continued for another six hours. Subjects' characteristics were recorded.

The two-tailed $P$ value equals 0.5784 . The value of the correlation coefficient $(R)$ is 0.5821 . This is a moderate positive correlation, which means there is a tendency for high $\mathrm{X}$ variable scores with high $\mathrm{Y}$ variable scores (and vice versa). The coefficient of determination (R2) is 0.3388 .

Information based measure of confidence was 0.16 , with a $95 \%$ confidence interval $(\mathrm{Cl})$ of 0.091 to 0.225 .

Interclass correlation (ICC) was 0.602 , with a $95 \% \mathrm{Cl}$ of 0.031 to 0.882 . 
Relationship curve between percentage of reduction and irradiance

A relationship curve between percentage of reduction and irradiance could be created based on percentage of reduction in serum bilirubin during the 0-6- and 0-12-hour interval if either linearity or proportionality is held to be true (Table 2, Figure 2). But such a graph is not possible if the relationship follows the saturation hypothesis due to lack of sufficient number of study points. Hypothetical values derived for both proportionality and linearity from the percentage of reduction in serum bilirubin during the 0-6- and 0-12-hour interval. $\square$

\section{DISCUSSION}

T his study was an attempt to find the relationship between irradiance and efficacy of phototherapy. In the first part of our research, we explored the possibility of whether the efficacy of phototherapy was predictable. Predicting efficacy is a difficult endeavour because there is a multitude of factors that have an influence on it. To nullify the effect of these factors, the predicted value was derived from the same subject to which the extrapolated value was applied to. For this, three time points were chosen for blood sampling viz. 0, 6 and 12 hours of phototherapy. The percentage of reduction in serum bilirubin during the 0-6-, 0-12- and 6-12-hour intervals was measured. The hypothesis was that the percentage of reduction during the 6-12-hour interval would be equal to that measured during the 0-6-hour interval if all experimental conditions were kept unchanged.

Both the actual and predicted values were compared by a battery of statistical test which found a strong correlation between the predicted and observed values. Interclass concordance was also found to be high, which shows that if the predicted value is high, the observed value also has the propensity to be high and vice versa.

Similar findings were reported by other authors. Thus, Schindl et al (14) found that the percentage of reduction in bilirubin levels measured at intervals of 2, 4, 6, 12, 18 and 24 hours was $9.4 \%, 16 \%, 23 \%, 40 \%, 44 \%$ and $50 \%$, respectively. If we calculate the percentage of reduction at individual time intervals of $0-2,2-4$ and 4-6 hours, the values come close to each other $(9.4 \%, 7.3 \%$ and $8.3 \%$, respectively). At $0-6$ and
6-12 hours, the percentage of reduction was $23 \%$ and $22 \%$, respectively. The aim of the quoted study was different, and its authors have not commented on this aspect. This was also the basis for chossing time points of 6 and 12 hours rather than 24 hours for bilirubin reduction used in other studies.

In the other group, irradiance was doubled after six hours and continued up to 12 hours. The predicted value was derived from the percentage of reduction at six hours. Three predicted values were calculated based on the two current theories on the relationship between efficacy and irradiance. One predicted value is based on considering a linear relationship and the other one on the existence of a saturation point. It should be noted that the study design used here will not be able to either prove or disprove or even detect a saturation point unless it has already been reached in the initial irradiance level (0-6-hour irradiance level). The third predicted value is based on the hypothesis, termed here as proportionality, that with increase in irradiance the efficacy will increase, but with diminishing returns. This calculated value also agrees with Bunson's law of reciprocity and fractional product method of drug agonism. Increase in efficacy (\% of reduction in bilirubin levels) will go on decreasing as a function of increase in irradiance level in terms of absolute decrease $(\mathrm{mg} / \mathrm{dL})$ but as a proportion it will remain constant. These three values were then compared with the actual measured percentage of decrease in serum bilirubin levels.

It was found that there was a significant difference between the values observed and those predicted based on linearity $(p=0.028)$. Data suggest that it is likely that the relationship between efficacy and irradiance is non-linear, which stands in contrast to the conclusion of Vandborg et al (12), who found a linear relationship. The quoted authors conducted their study on four different groups with different irradiance levels. Although they did not get a linear relationship with all groups, a rather linear relationship was found on merging the data of middle two groups (with intermediate irradiance level). It could be argued that their conclusion may not have been the most appropriate one. A logical inconsistency also stems from the fact that, if the linear relationship line is extended to higher values, at a certain value of irradiance the percentage of reduction in bilirubin levels would exceed $100 \%$, which would be phy- 
sically impossible. Thus, there could be a part of relationship that could satisfy linearity but would not be able to explain the whole relationship.

The difference between values predicted by proportionality and actual observed ones was found to be not significant. Therefore, proportionality as a hypothesis could not be rejected according to the results obtained in this study. It also showed the highest value for interclass concordance among all groups and indicated that the actual values moved closest with those for proportionality rather than with linearity or saturation. The disagreement between predicted and observed values was also found to be lowest for values predicted by proportionality.

Comparing actual observed values with those predicted for saturation reveals a statistically significant difference $(p=.0017)$. Interclass concordance was also found to be the lowest and disagreement measure was the highest among the three groups.

It could be noted that the correlation coefficient and the coefficient of determination were high for all groups of predicted values because all values were derived from the observed reduction during the 0-6-hour interval. Since all values had a single source value, they were bound to have a similar correlation to actual observed values. The high correlation also suggests that the percentage of reduction in bilirubin levels during the 6-12-hour interval is closely dependent on the decrease during the 0-6-hour interval. This is also supported by results of the first group in this study as well as those reported by Carvalho et al (14). Moreover, the high correlation suggests a possible relationship between two variables and it is in no way a measure of agreement between them.

\section{CONCLUSION}

Tre he present study suggests that the relationship between efficacy, as measured by percentage of reduction in serum bilirubin, and irradiance is unlikely to be linear. Data are insufficient to clearly distinguish between proportionality and saturation point, considering that the results may be possible with both hypotheses. Even though laws of photochemistry do not support the existence of a saturation point, the study design used by us is incapable of refuting it with any degree of certainty. Predictability was found to be possible if sufficient initial information is available and experimental conditions are kept constant. Moreover, it seems that there is no direct relationship, but only a correlation, between efficacy and irradiance per se, but there will be a causal relationship between efficacy and energy dose. Our novel study design was found to be practicable and usable for further studies, as it could also be modified for different research question. Further study with a higher sample size is required to adequately elaborate the relationship between efficacy and irradiance.

Conflicts of interest: none declared. Financial support: none declared.

\section{R}

1. Deorari AK, Chellani H, Carlin JB, et al. Clinicoepidemiological profile and predictors of severe illness in young infants (<60 days) reporting to a hospital in North India. Indian Pediatr 2007;44:739-748.

2. Dennery PA. Pharmacological interventions for the treatment of neonatal jaundice. Semin Neonatol 2002;7:111-119.

3. Watchko JF, Daood MJ, Hansen TW. Brain bilirubin content is increased in P-glycoprotein-deficient transgenic null mutant mice. Pediatr Res 1998;44:763-766.

4. Brouillard RP. Measurement of red blood cell life-span. JAMA 1974;230:1304-1305.

5. Leakey JE, Hume R, Burchell B. Development of multiple activities of UDP- glucuronyltransferase in human liver. Biochem J 1987;243:859-861.
6. Strassburg CP, Strassburg A, Kneip S, et. al. Gut developmental aspects of human hepatic drug glucuronidation in young children and adults. Gut 2002;50:259-265.

7. Fevery J. Bilirubin in clinical practice: a review. Liver Int 2008;28:592-605.

8. Bonnett R, Davies JE, Hursthouse MB, Sheldrick GM. The structure of bilirubin. Proc R Soc Lond B Biol Sci 1978;202:249-268.

9. Ostrow JD, Mukerjee P, Tiribelli C. Structure and binding of unconjugated bilirubin: relevance for physiological and pathophysiological function. J Lipid Res 1994;35:1715-1737.

10. Ip S, Chung M, Kulig J, et.al. American Academy of Pediatrics Subcommittee on Hyperbilirubinemia. An evidence-based review of important issues concerning neonatal hyperbilirubinemia. Pediatrics 2004;114:e130-e153.

11. Tan KL. The nature of the dose-response relationship of phototherapy for neonatal hyperbilirubinemia. J Pediatr 1977;90:448-452.

12. Vandborg PK, Hansen BM, Greisen G, Ebbesen F. Dose-Response Relationship Phototherapy for Hyperbilirubinemia. Pediatrics 2012;130:352-357.

13. Schindl A, Rosado-Schlosser B, Trautinger F. Die Reziprozitätsregel in der Photobiologie. Eine Ubersicht [Reciprocity regulation in photobiology. An overview]. Hautarzt 2001;52:779-785.

14. de Carvalho M, Mochdece CC, Sá CA, Moreira ME. High-intensity phototherapy for the treatment of severe nonhaemolytic neonatal hyperbilirubinemia. Acta Paediatr 2011;100:620-623. 